\title{
A CRITIQUE FOR SHALLOW WATER THEORY
}

\author{
by A. G. MACKIE \\ (Received 17th August 1983)
}

\section{Introduction}

There is now a huge volume of literature dealing with exact and approximate solutions of the equations governing the motion of an inviscid, incompressible fluid. Major difficulties in finding exact solutions arise when the fluid is bounded by a socalled "free" surface on which the pressure is constant because the equation of such a surface is not known in advance. A boundary condition of this type arises when a mathematical analysis of water wave theory is attempted. Different approximations are, of course, appropriate to different circumstances and here we deal with a well known approximation for waves in shallow water which will be described in detail in the next section. The main ideas underlying this particular approximation have been known for some time but it is probably true to say that they were first brought into real prominence by Stoker [3] in a long article in the Communications of Pure and Applied Mathematics. The main ideas in this article were later developed in considerable detail in Stoker's book "Water Waves" [4]. More recently this approximation has been used by Sewell and Porter [2] as one of several examples in fluid and solid mechanics which are given a geometrical interpretation in terms of "constitutive surfaces" which has close links with catastrophe theory. An excellent account of this interesting approach, which lists many associated references, is given by Sewell [1].

Shallow water theory is a very attractive theory, not least because the equations which arise in it are formally identical with the exact equations governing the flow of a perfect polytropic gas of adiabatic index 2 . This means that many well known results in gas dynamics can be taken over directly and given an interpretation in terms of long waves in shallow water. There is always the danger that the very elegance of a mathematical model will tempt research workers to apply it to problems without adequate consideration of whether the assumptions inherent in the model are any longer appropriate.

The object of this present paper is to seek a critique for establishing the validity of the shallow water approximation when used in particular cases. The approach used, however, has several interesting byproducts. It offers a physical and geometrical picture of the analogy between the water wave and gas dynamic interpretations. It also enables certain anomalies which have occasionally surfaced in the literature to be easily explained.

\section{The hydrostatic pressure assumption and the resulting equations}

We consider an inviscid fluid of constant density $\rho$ moving in the $x, y$-plane with velocity components $u(x, y, t), v(x, y, t)$, where $t$ is the time. The $y$-axis points vertically 
upwards and gravity forces act. For convenience and simplicity we restrict consideration to two dimensions but the results can easily be extended to three-dimensional flow. The fluid is bounded from below by the (usually prescribed) surface $y=f(x, t)$ and from above by the unknown free surface $y=\eta(x, t)$ on which the pressure $p$ is constant, this constant usually being taken to be zero. In practice $f(x, t)$ is normally independent of $t$ and is frequently taken to be zero, the case of a flat horizontal bottom.

The fundamental assumption in the shallow water approximation considered in this paper is that the pressure is hydrostatic. This means that the dynamic contribution to the pressure arising from the motion of the fluid is ignored. Thus

$$
p=\rho g(\eta(x, t)-y)
$$

where $g$ is the acceleration due to gravity.

It is now necessary to ask under what circumstances $(2.1)$ is a reasonable assumption to make and how good an approximation to the full equations are the subsequent equations derived on this assumption. One common practice is to make some rather vague statements about horizontal scales being large compared with vertical scales or to say that the approximation is likely to be reasonable for waves which are long compared with the mean depth of the fluid. A mathematically more sophisticated approach is to seek an expansion of the equations of motion in terms of some parameter extracted from a suitable combination of rescaled variables. The vulnerability of this approach is not lost on the acknowledged authorities. Thus Wehausen and Laitone [5] state "As used here the method is purely formal, the nature of the convergence of the perturbation series, whether it be uniform, pointwise, asymptotic or what not, being left open." In similar vein Stoker [4, p. 31] remarks "However it is not of course true that we have proved that $(2.4 .17)$ [(2.1) in the notation of this paper] is in some sense an appropriate assumption: instead, it should be admitted frankly that our dimensionless variables were introduced in just such a way that (2.4.17) would result".

The point of view adopted in the present paper is markedly different. No attempt is made to set up a series expansion and the intricacies of defending the resulting convergence or otherwise of such an expansion are thereby avoided. The main thrust of the argument is as follows. Any solution of the approximating set of equations may be regarded as a solution of the full Euler equations of motion provided some additional fictitious forces are assumed to act. The magnitude of these fictitious forces, when compared with that of the actual forces which cause the motion, should provide some measure of the validity of the approximating assumption, bearing in mind the wellposed nature of the boundary value problems considered.

We first treat the case where the fluid is bounded from below by the flat horizontal bottom $y=0$. The equations of motion are

$$
\begin{aligned}
& \frac{D u}{D t} \equiv \frac{\partial u}{\partial t}+u \frac{\partial u}{\partial x}+v \frac{\partial u}{\partial y}=-\frac{1}{\rho} \frac{\partial p}{\partial x} \\
& \frac{D v}{D t} \equiv \frac{\partial v}{\partial t}+u \frac{\partial v}{\partial x}+v \frac{\partial v}{\partial y}=-\frac{1}{\rho} \frac{\partial p}{\partial y}-g
\end{aligned}
$$


together with the continuity equation

$$
\frac{\partial u}{\partial x}+\frac{\partial v}{\partial y}=0
$$

The boundary conditions are $v=0$ on $y=0$ and $p=0$ on $y=\eta(x, t)$. In addition, since the upper bounding surface consists of the same fluid particles,

$$
\frac{D \eta}{D t} \equiv \frac{\partial \eta}{\partial t}+u \frac{\partial \eta}{\partial x}=v
$$

on $y=\eta(x, t)$. We now suppose that (2.1) holds exactly. Then (2.2) yields

$$
\frac{D u}{D t}=-g \frac{\partial \eta}{\partial x}
$$

We now make a final assumption that at some time, say $t=0, u$ does not depend on $y$. Then, since the right hand side of (2.6) does not depend on $y, u$ in the subsequent motion is a function of $x$ and $t$ only. Equation (2.6) then reduces to

$$
\frac{\partial u}{\partial t}+u \frac{\partial u}{\partial x}+g \frac{\partial \eta}{\partial x}=0
$$

If $(2.4)$ is integrated with respect to $y$, then

$$
v=-y \frac{\partial u}{\partial x}
$$

since $v=0$ when $y=0$, and when this is applied on $y=\eta(x, t)$, using (2.5),

$$
\frac{\partial \eta}{\partial t}+u \frac{\partial \eta}{\partial x}+\eta \frac{\partial u}{\partial x}=0
$$

Equations (2.7) and (2.9) are the usual equations of shallow water theory. If functions $u(x, t), \eta(x, t)$ are found which satisfy them, then $u$, together with $p$ and $v$ as determined by (2.1) and (2.8) respectively, exactly satisfy the boundary conditions and the set of equations (2.2)-(2.4) with the exception of (2.3). If, however, an extra force $G(x, y, t)$ per unit mass is presumed to act in the vertical direction and is therefore added to the right hand side of (2.3), we see that this revised equation too is now exactly satisfied provided

$$
G(x, y, t)\left(=\frac{D v}{D t}\right)=-y\left\{u \frac{\partial^{2} u}{\partial x^{2}}+\frac{\partial^{2} u}{\partial x \partial t}-\left(\frac{\partial u}{\partial x}\right)^{2}\right\}
$$

when use is made of (2.8). 


\section{Implications of new approach}

It is now possible to derive the well known analogy with gas dynamics but we shall do rather more than just formally verify the equivalence of two pairs of equations. As the fluid is incompressible, its mass per unit area is constant but the mass per unit length along the $x$-axis is not. The mass contained in a vertical slice of thickness $d x$ is $\rho \eta d x$ and this leads us to define

$$
\bar{\rho}=\rho \eta
$$

as a line density. The total pressure force acting on the left hand slice is

$$
\bar{p}=\int_{0}^{\eta} p d y=\frac{1}{2} \rho g \eta^{2}
$$

using (2.1), and this, with (3.1), gives

$$
\bar{p}=\frac{g}{2 \rho} \bar{\rho}^{2} .
$$

When we consider the horizontal forces acting on this vertical slice we see that they come solely from the gradient of the pressure $\bar{p}$ because the fictitious force is vertical, the reaction from the flat bottom is vertical and there is no force on the curved upper boundary since $p=0$ there. The analogy with one-dimensional gas dynamics is thus complete and we can write down the momentum equation

$$
\frac{\partial u}{\partial t}+u \frac{\partial u}{\partial x}=-\frac{1}{\bar{\rho}} \frac{\partial \bar{p}}{\partial x}
$$

together with the continuity equation

$$
\frac{\partial \bar{\rho}}{\partial t}+\frac{\partial}{\partial x}(\bar{\rho} u)=0
$$

These equations are, of course, identical with (2.7) and (2.9) when (3.1) and (3.2) are used but why this is so is now physically obvious.

When $G(x, y, t)$, as given by (2.10), is zero, any solution of (2.7) and (2.9) is an exact solution of the full equations of motion for a fluid bounded by a rigid horizontal bottom and a free surface $y=\eta(x, t)$ on which $p=0$. An obvious question to ask is whether any such exact solutions do exist. To examine this we first consider the vorticity

$$
\zeta=\frac{\partial v}{\partial x}-\frac{\partial u}{\partial y}
$$

which, from (2.8), reduces to 


$$
\zeta=-y \frac{\partial^{2} u}{\partial x^{2}}
$$

The convective rate of change of vorticity is given by

$$
\frac{D \zeta}{D t}=\frac{\partial G}{\partial x}
$$

after some manipulation. When $G=0$ this is a restatement of Kelvin's vorticity theorem. If this flow is to be irrotational, then from (3.4)

$$
u(x, t)=u_{0}(t)+k(t) x
$$

and to satisfy $G=0$, using (2.10),

$$
k^{\prime}(t)=\{k(t)\}^{2}
$$

which means that

$$
u(x, t)=u_{0}(t)-\frac{x}{t+t_{0}}
$$

where $t_{0}$ is a constant. Substituting this into (2.7) we obtain, after one integration,

$$
g \eta(x, t)=w(t)-\left\{u_{0}^{\prime}(t)+\frac{u_{0}(t)}{t+t_{0}}\right\} x-\frac{x^{2}}{\left(t+t_{0}\right)^{2}}
$$

It is now easy to verify that $u$ and $\eta$ as given by (3.6) and (3.7) cannot, for any finite value of $t_{0}$, satisfy equation (2.9). As $t_{0} \rightarrow \infty$ we obtain a family of solutions which has some features of interest. Now $u=u_{0}(t)$ and $g \eta=w(t)-u_{0}^{\prime}(t) x$. For these to satisfy (2.9), $u_{0}^{\prime \prime}(t)=0$ and $w^{\prime}(t)=u_{0}(t) u_{0}^{\prime}(t)$. This gives

$$
\begin{aligned}
u(x, t) & =A t+B \\
g \eta(x, t) & =\frac{1}{2}(A t+B)^{2}+C-A x
\end{aligned}
$$

where $A, B, C$ are arbitrary constants. With $A=0$ we recover the obvious trivial solution $u=$ constant, $\eta=$ constant. With $A \neq 0$ we can choose $B=C=0$ without any essential loss of generality. The motion thus obtained is that of an infinite wedge of fluid, initially at rest and occupying the region $x<0,0<y<-A x / g$, moving to the right for $t>0$ with constant acceleration $A$.

With these exceptions there are no irrotational flows which satisfy the full equations of motion and also the shallow water equations. This is disappointing but hardly surprising. The above discussion does, however, prompt the following observation. Shallow water theory has sometimes been criticised because the solutions it offers are 
not irrotational. The criticism is a naive one since any failure to meet the full equations is liable to provide solutions with properties other than those of solutions of the exact equations but the present approach pinpoints the nature of the deficiency in this case with precision. The shallow water equations are exact if the fictitious forces are deemed to act and these fictitious forces are, in general, non-conservative. Thus vorticity will be generated and in a manner given analytically by (3.5).

Ideally we would like, for a given solution of (2.7) and (2.9), to evaluate the fictitious force $\rho G(x, y, t)$ as given by (2.10) and compare it with the actual external body force $\rho g$. In practice this is very difficult since, in the first place, very few exact analytical solutions of (2.7) and (2.9) are known and, for those which are, the evaluation of second derivatives is a task of daunting complexity. One of the few examples where a tractable solution is available is the case of the point-centred simple wave. Physically fluid is at rest at constant height $H$ in the region $x>0$ when, at $t=0$, the vertical barrier bounding it at $x=0$ is instantaneously removed. At a subsequent time $t$, the liquid occupies the region $x>-2 c_{0} t$, where $c_{0}^{2}=g H$, and the solution is given by

$$
\begin{gathered}
u=\frac{2}{3}\left(\frac{x}{t}-c_{0}\right), \eta=\frac{1}{9 g}\left(\frac{x}{t}+2 c_{0}\right)^{2},\left(-2 c_{0} t<x<c_{0} t\right) \\
u=0, \eta=H . \quad\left(x \geqq c_{0} t\right)
\end{gathered}
$$

In (3.8) we evaluate $G(x, y, t)$, using (2.10), to get

$$
G(x, y, t)=-\frac{10 y}{9 t^{2}}
$$

and hence the approximate solution can be deemed good if $t \gg(H / g)^{1 / 2}$. Of course the singular nature of the solution at $t=0$ makes the analysis meaningless for small values of $t$. Perhaps the best way to interpret this problem is to postulate an initial value problem starting at some $t=t_{0} \gg(H / g)^{1 / 2}$ with initial conditions given by (3.8) and (3.9) where $t$ is replaced by $t_{0}$. For values of $t>t_{0}$, (3.8) and (3.9) then give the exact solution of the shallow water equations and represent an excellent approximation to a solution of the full equations since $|G / g| \ll 1$.

The above example highlights the problem of how precisely to interpret $|G|<|g|$. A pointwise comparison is clearly not satisfactory. Moreover the hyperbolic nature of the system means that any discontinuities in the derivatives of the initial data will be propagated along the characteristics which are the curves obtained by integrating

$$
\frac{d x}{d t}=u \pm(g \eta)^{1 / 2}
$$

Thus the second order derivatives in (2.10) may not exist at all points and some sort of distributional approach may be required. We do not pursue this further here but use the introduction of this aspect to demonstrate how the fictitious force approach explains the apparent paradox wherein small disturbances in a fluid which is defined to be 
completely incompressible apparently propagate at a finite speed. Attention was drawn to this by Friedrichs in an appendix to Stoker's paper [3] through the remark

"The problem is of considerable mathematical interest also because of the following intriguing circumstance: The approximation of lowest order to the solution of a potential problem is sought in the form of a solution of a nonlinear wave problem, and this means that the solution of a problem of elliptic type is approximated, at least in the lowest order, by the solution of a problem of hyperbolic type."

The concept of fictitious forces throws considerable light on this matter. First it should be observed in relation to the comment by Friedrichs that it would be more appropriate to call the problem of finding a solution of the full system of equations one of parabolic rather than elliptic type. It is true that the velocity potential in irrotational flow must satisfy Laplace's equation as a function of $x$ and $y$ but since in unsteady flow the potential is a function of $t$ as well as of $x$ and $y$, this equation should formally be classified as parabolic in the broad sense rather than elliptic. This, however, does not explain the finite propagation speed since, even in parabolic problems, disturbances propagate with infinite speed. The following simplified model indicates the essence of the situation as it occurs in shallow water theory.

We consider the equation

$$
\frac{\partial^{2} \phi}{\partial x^{2}}-\frac{\partial \phi}{\partial t}=s(x, t) \quad(-\infty<x<\infty)
$$

and the initial condition $\phi=l(x)$ when $t=0$ where the functions $l(x)$ and $s(x, 0)$ are zero when $|x|>1$ and are continuously differentiable as often as required for all $x$. With $s(x, t)$ regarded as known, this equation is formally parabolic and may be thought of as representing the temperature distribution in an infinite rod with a certain time and space dependent distribution of heat sources and a given initial temperature where this source distribution and temperature vanish when $t=0$ for $|x|>1$. In general we would expect that, for arbitrarily small $t>0$, the effects of the "disturbance", that is of the nonzero values of $\phi(x, 0)$ in $|x|<1$, would have propagated to all points of the rod. No finite propagation speed can be identified.

We now consider the equation

$$
\frac{\partial^{2} \phi}{\partial x^{2}}-\frac{\partial \phi}{\partial t}=\frac{\partial^{2} \phi}{\partial t^{2}} \quad(-\infty<x<\infty)
$$

with the initial conditions $\phi=l(x), \partial \phi / \partial t=0$, the function $l(x)$ being the same as above. This may be interpreted as the equation governing the transverse displacement of a string initially at rest when the initial displacement is zero for $|x|>1$ and prescribed for $|x|<1$, the subsequent motion being linearly damped. It is not difficult to solve this problem explicitly. We denote the solution by $\phi_{0}(x, t)$ and observe the essential property that $\phi_{0}(x, t)$ is zero when $|x|>t+1$, indicative of the physical fact that the disturbance propagates out at finite (in this case unit) speed. We now obtain $\partial^{2} \phi_{0} / \partial t^{2}$ as a function of $x$ and $t$, set it equal to $s(x, t)$ and examine the boundary value problem posed above for equation (3.10) with this particular choice of $s(x, t)$. Clearly the solution of this 
problem is $\phi_{0}(x, t)$, a solution that propatgates at finite speed despite the fact that the governing equation is parabolic. The role played by $\partial^{2} \phi_{0} / \partial t^{2}$ in the above model is essentially that played by the fictitious force $\rho G(x, y, t)$ in shallow water theory. When $G(x, y, t)$ is added to the right hand side of (2.3), the boundary value problem represented by (2.1)-(2.4) with the given boundary and appropriate initial conditions, including the condition that $u$ is independent of $y$ when $t=0$, can be completely identified with a boundary value problem for the system (2.7) and (2.9). The latter is a hyperbolic system whereas the former is, at least formally, a parabolic system.

We conclude this section by examining briefly the behaviour of solutions of the equation obtained by setting $G(x, y, t)=0$, that is solutions of

$$
u \frac{\partial^{2} u}{\partial x^{2}}+\frac{\partial^{2} u}{\partial x \partial t}-\left(\frac{\partial u}{\partial x}\right)^{2}=0
$$

This is a second order, quasi-linear, partial differential equation. Routine analysis shows that the equation is hyperbolic and that the two families of characteristics are respectively the lines $t=$ constant and the particle paths obtained by integrating

$$
\frac{d x}{d t}=u
$$

The compatibility conditions along the latter family are integrable and yield

$$
t+\left(\frac{\partial u}{\partial x}\right)^{-1}=\text { constant }
$$

This indicates that $\partial u / \partial x \rightarrow 0$ as $t$ increases. Detailed analysis is, of course, pointless since no exact solutions exist which are also solutions of (2.7) and (2.9) outside the very limited class found earlier but it is nevertheless curious that such an analysis is feasible.

\section{Non-horizontal lower boundary}

When the fluid is bounded from below by the surface $y=f(x, t)$ instead of $y=0$ which was the assumption made in Sections 2 and 3, much of the analysis can be appropriately generalised. The boundary condition on the bottom is now

$$
v=\frac{\partial f}{\partial t}+u \frac{\partial f}{\partial x}
$$

when $y=f$ so that when (2.4) is integrated with respect to $y$ we obtain

$$
v=\frac{\partial f}{\partial t}+u \frac{\partial f}{\partial x}-(y-f) \frac{\partial u}{\partial x}
$$


On $y=\eta, v$ is still given by (2.5) and so, when this is combined with (4.1),

$$
\frac{\partial h}{\partial t}+u \frac{\partial h}{\partial x}+h \frac{\partial u}{\partial x}=0
$$

where $h(x, t)=\eta-f$ is the height of the vertical column of fluid at position $x$ and time $t$. Equation (2.7) is unaffected by the sloping bottom but since $h$ is the new dependent variable it is rewritten as

$$
\frac{\partial u}{\partial t}+u \frac{\partial u}{\partial x}+g \frac{\partial h}{\partial x}=-g \frac{\partial f}{\partial x}
$$

When the shape of the bottom is known, the right hand side of (4.2) is also known. The analogy with gas dynamics then goes through as before except that the associated gas now moves under the action of a known external force as well as its own pressure gradient. An interesting special case is when the bottom is a plane inclined to the horizontal. The analogous problem in gas dynamics is that of a gas moving in a tube under the action of a constant force such, for example, as gravity. Moreover, if we consider the horizontal equation of motion of a vertical slice of the fluid as at the beginning of Section 3 we can readily see where this force comes from. Because the bottom is sloping, its reaction on the fluid slice has a horizontal component and since the fluid pressure at the bottom is $\rho g h$ this can be identified precisely with the right hand side of (4.2).

The fictitious forces are still in the vertical direction only but are now much more complicated because of (4.1) replacing (2.8) as the expression for $v$. To avoid excessive algebra we restrict attention to the most commonly encountered case when $f$ is independent of $t$, that is when the sloping bottom is fixed or rigid. The fictitious force per unit mass is then given by

$$
G(x, y, t)=-(y-f)\left\{u \frac{\partial^{2} u}{\partial x^{2}}+\frac{\partial^{2} u}{\partial x \partial t}-\left(\frac{\partial u}{\partial x}\right)^{2}\right\}-g \frac{d f}{d x} \frac{\partial \eta}{\partial x}+u^{2} \frac{d^{2} f}{d x^{2}}
$$

On the assumption that the bottom does not slope too steeply, it would seem that the deficiencies of the approximating system are no greater for a sloping than for a flat bottom although the presence of the last term on the right hand side of (4.3) gives a warning that neither the speed of the fluid nor the curvature of the bottom must be too great. More precisely and with proper regard to maintaining dimensional accuracy, the speed squared times the curvature should remain small compared with $g$.

\section{The hydraulic analogy for a compressible fluid}

Some of the previous results can be generalised to the case where the original fluid, instead of being incompressible, has variable density $\rho$ which is a single-valued function of the pressure $p$ and denoted by $\rho(p)$. This generalisation has certain features of theoretical interest and may have some application in the study of stratified flows. The 
momentum equations (2.2) and (2.3) are unchanged but the continuity equation (2.4) is now

$$
\frac{\partial \rho}{\partial t}+\frac{\partial(\rho u)}{\partial x}+\frac{\partial(\rho u)}{\partial y}=0
$$

The hydrostatic pressure is obtained by solving

$$
\frac{d p}{d y}=-g \rho(p)
$$

subject to the condition $p=0$ when $y=\eta$. We now make the assumptions that this pressure distribution holds in the moving fluid also and that at some initial time $u$ is a function of $x$ only. From (5.2) it follows that

$$
\int_{0}^{p} \frac{d p}{\rho(p)}=g(\eta(x, t)-y)
$$

and hence that $\rho^{-1} \partial p / \partial x$ is a function of $x$ and $t$ only. From (2.2), $u$ will continue to be independent of $y$ as before. A gas dynamics analogy therefore exists as in the case of constant density with $\bar{\rho}, \bar{p}$ defined by

$$
\bar{\rho}=\int_{0}^{\eta} \rho(y) d y \quad \text { and } \quad \bar{p}=\int_{0}^{\eta} p(y) d y
$$

It will be noted that only the case of the flat horizontal bottom is treated here. From (5.3), $p$ is a function of $\eta-y$ and consequently the density is also a function of $\eta-y$. We denote this by $\rho=r(\eta-y)$. Then (5.4) becomes

$$
\bar{\rho}=\int_{0}^{\eta} r(y) d y \quad \text { and } \quad \bar{p}=g \int_{0}^{\eta}(\eta-y) r(y) d y
$$

The relationship between $\bar{p}$ and $\bar{\rho}$ is obtained by eliminating $\eta$ from the two equations in (5.5).

The fictitious force $\rho G(x, y, t)$ which is necessary to preserve the exact equivalence of the two sets of equations is again associated purely with the vertical momentum equation (2.3). If $u(x, t)$ is known, $v(x, y, t)$ is found by integrating (5.1) with respect to $y$ and then $G(x, y, t)=D v / D t$. The details are omitted.

When the original $p, \rho$ relationship is that of a polytropic gas so that $p \propto \rho^{\gamma}$, $r(y) \propto y^{1 /(\gamma-1)}$. Substitution in (5.5) leads, after some simplification, to

$$
\bar{p} \propto \bar{\rho}^{\bar{\gamma}} \quad \text { where } \quad \bar{\gamma}=\frac{2 \gamma-1}{\gamma} .
$$

The classical case in which $\bar{\gamma}=2$ (see (3.3)) arises in incompressible flow as a limiting case as $i \rightarrow \infty$. Equation (5.2) cannot be integrated with the given boundary condition 
unless $\gamma>1$ but this property is inherent in all real polytropic gases anyway. In summary an analogy exists for any polytropic gas with adiabatic index $\gamma>1$ in terms of a second polytropic gas of adiabatic index $\bar{\gamma}, \gamma$ and $\bar{\gamma}$ being related by (5.6).

It is possible to modify the case $\gamma=1$ by considering the pressure, density relationship

$$
p=c^{2}\left(\rho-\rho_{0}\right)
$$

where $c, \rho_{0}$ are constants and $\rho \geqq \rho_{0}$. This is a good model for a nearly incompressible fluid which takes compressibility into account to the first order. Integration of (5.2) gives

$$
\rho=\rho_{0} e^{g(\eta-y) / c^{2}}
$$

and from (5.5)

$$
\begin{gathered}
\bar{\rho}=\frac{\rho_{0} c^{2}}{g}\left(e^{g \eta / c^{2}}-1\right), \\
\bar{p}=\frac{\rho_{0} c^{4}}{g}\left(e^{g \eta / c^{2}}-1-\frac{g \eta}{c^{2}}\right) .
\end{gathered}
$$

Thus the motion under gravity of a slightly compressible fluid with an equation of state described by (5.7) bounded by a free surface and a flat bottom is analogous to the onedimensional motion of a gas with an equation of state obtained by eliminating $\eta$ from (5.9) and (5.10). The familiar forms appropriate to a strictly incompressible fluid are readily obtained by letting $c \rightarrow \infty$ in equations (5.7)-(5.10).

\section{REFERENCES}

1. M. J. Sewell, Legendre transformations and extremum principles, The Rodney Hill 60th Anniversary Volume (Pergamon Press, Oxford, 1981).

2. M. J. Sewell and J. Porter, Constitutive surfaces in fluid mechanics, Math. Proc. Camb. Phil. Soc. 88 (1981), 517-546.

3. J. J. Stoker, The formation of breakers and bores, Comm. Pure Appl. Math. 1 (1948), 1-87.

4. J. J. Stoker, Water Waves (Interscience, New York, 1957).

5. J. V. Wehausen and E. V. Laitone, Surface waves, Handbüch der Physik 9 (1960), 446-778.

UNIVERSITY OF EDINBURGH 